\title{
Genetic Variability and Traits Association Analysis of Tomato (Lycopersicon esculentum L.) Genotypes for Yield and Quality Attributes
}

\author{
Md. Mehedi Hasan, Md. Abdullah Al Bari and Mohammad Anwar Hossain* \\ Department of Genetics and Plant Breeding, Bangladesh Agricultural University, Bangladesh \\ *Corresponding Author: anwargpb@bau.edu.bd
}

Copyright $(2016$ by authors, all rights reserved. Authors agree that this article remains permanently open access under the terms of the Creative Commons Attribution License 4.0 International License

\begin{abstract}
The experiment was conducted at the experimental farm of the department of Genetics and Plant Breeding, Bangladesh agricultural university, Mymensingh, Bangladesh, with 30 different tomato (Lycopersicon esculentum L.) genotypes to study the genetic variability and characters association for yield and yield-contributing traits along with quality traits. The experiment was laid out in a Randomized Complete Block Design with three replications. Analysis of variances showed high degree of variation existed among the genotypes of the studied traits. Yield contributing traits showed higher phenotypic co-efficient of variation as compared to their corresponding genotypic co-efficient of variation indicating considerable environmental influences on them. Individual fruit weight showed high heritability (99.71) with high genetic advance (85.4) followed by fruits per plant (99.65 \& 81.26) suggested a good scope for the improvement of these characters through selection. High heritability but low genetic advance showed in number of primary branches (75.87 \& 3.67), fruit cluster per plant (99.49 \& 6.12), fruit diameter (98.83 \& $5.59)$, total soluble solid (80.51 \& 1.85) and ascorbic acid content (90.75 \& 9.52) indicated limited scope for the improvement of these traits through selection. Correlation coefficient analysis showed that yield per plant had significant positive correlations with individual fruit weight $\left(0.609^{* * *}\right)$ and number of fruit cluster per plants $\left(0.396^{*}\right)$ whereas significant negative correlation was observed with number of primary branches per plant $\left(-0.421^{*}\right)$. Path
\end{abstract}

co-efficient analysis revealed that the maximum positive direct contribution towards yield was through individual fruit weight $(0.704)$ whereas plant height (-0.038) showed negative direct contribution towards yield. Individual fruit weight and number of fruit cluster per plant could be crucial for improving tomato yield.

Keywords Tomato, Genetic Diversity, Heritability, Genetic Advance, Correlation co-efficient, Path co-efficient, Yield, Quality

\section{Introduction}

The cultivated tomato (Lycopersicon esculentum L.) occupies the prime position among different vegetables and grown in a variety of climatic condition all over the world. It belongs to the family Solanaceae with chromosome number of 24 and is a self-pollinated, annual crop [1]. The position of tomato in world vegetable production is second only after potato [2]. It is a rich source of vitamin A, C (ascorbic acid, AsA), glutathione (GSH) and mineral nutrients with preponderance of $\mathrm{Ca}, \mathrm{P}$ and $\mathrm{Fe}$ [3]. Tomato fruit and its products are the main source of lycopene and other antioxidants in the human diet [4]. The level of lycopene in tomato fruit increases 500 times during ripening [5]. High antioxidant capacity in both fresh and processed tomatoes associated with the higher capacity to eliminate reactive oxygen species (ROS) and helps in lowering the incidence of 
certain forms of human cancer [6]. In many countries it is considered as “poor man's orange" because of its improved nutritional values [7]. Recent epidemiological studies have shown that their consumption helps to prevent cardiovascular disease [8,9] and some types of cancers, such as prostate cancer $[10,11]$.

Tomato like other crops is the final product attributed by a complex chain of interrelating effects of different characters $[12,13]$. The degree of association between characters as indicated by the correlation coefficients has always been a helpful instrument for the selection of desirable characters in a breeding program. Correlation coefficient analysis measures the mutual relationship between various plant characters and determines the component characters on which selection can be based for yield improvement. Correlation coefficient is not adequate to anticipate traits interrelationship leading to yield. Under such circumstances, path coefficient analysis is an additional informative tool $[12,14]$. Path analysis splits the correlation coefficients into direct and indirect effects of a set of dependent variables on the independent variables which help in selecting elite genotypes. The first and foremost prerequisite for crop improvement is the availability of wide genetic variability in the form of germplasm [15]. The influence of environment in expression of characters and the extent to which improvement is possible after selection can be determined by heritability and genetic advance [16]. Heritable variation can be effectively studied in conjunction with genetic advance. High heritability alone is not enough to make efficient selection in segregating generation and needs to be accompanied by a substantial amount of genetic advance [17]. Amounts of variability present in germplasm can be detected by phenotypic and genotypic coefficients of variation.

In Bangladesh there are nearly 30 released tomato varieties and 5 tomato hybrids developed by Bangladesh Agricultural Research Institute (BARI) and Bangladesh Institute of Nuclear Agriculture (BINA) and other private seed companies. However, information on the quality attributes like antioxidant compounds in the released varieties is unexpectedly rare. Additionally, in Bangladesh majority of the Asian Vegetable Research and Development Center (AVRDC) germplasm has not been characterized for their yield and antioxidant properties. Importantly, we need to characterize them under the climatic conditions of Bangladesh for determining potential genotypes that can be used for future plant breeding program to improve yield and quality. The present investigation was therefore undertaken to study the genetic variability and character association among 30 diverse tomato genotypes for yield and quality attributes.

\section{Materials and Methods}

The experiment was conducted during the period from November 2014 to April 2015 in the experimental farm of the Department of Genetics and Plant Breeding, Bangladesh Agricultural University, Mymensingh. The experiment was laid out in RCBD with three replications. This study involved 14 exotic (V1007282, V1006136, V1006422, V1006186, V1006484, V1045786, V1063607, WP7, WP8, WP10, C11, C41, C51, C71), 2 indigenous advance line (Phili-1, Phili-2) and 14 varieties (BARI tomato-9, BARI tomato-11, BARI tomato-14, BARI tomato-15, BARI tomato-8,BINA tomato-2, BINA tomato-7, BINA tomato-3, BINA tomato-4, BINA tomato-5, BINA tomato-8, BINA tomato-9, BINA tomato-10) of tomato. The exotic tomato lines were collected from AVRDC, Taiwan. The indigenous tomato lines and variety were collected from Bangladesh Agricultural Research Institute (BARI), Gazipur and Bangladesh Institute of Nuclear Agriculture (BINA), Mymensingh. Data on 14 different characters i.e. i) plant height $(\mathrm{cm})$, ii) number of primary branches per plant, iii) days to first flowering, iv) days to $50 \%$ flowering, v) number of fruit cluster per plant (no.), vi) days to first harvest, vii) individual fruit weight $(\mathrm{g})$, viii) fruit diameter $(\mathrm{cm}), \mathrm{ix})$ fruits per plant (no.), x) Days to last harvest, xi) Yield per plant $(\mathrm{kg})$, xii) dry matter content (\%), xiii) determination of total soluble solid (TSS) of tomato (\%), xiv) ascorbic acid (AsA) content $(\mathrm{mg} / 100 \mathrm{~g})$ were recorded for the study. Data were collecting from three replications for each genotype and five randomly selected plants from each replication. Mean values for each character were assembled and used for statistical analysis. AsA content was determined according to the method described by Plummer [18].

Analysis of variance was performed using the statistical software SAS. Genotypic and phenotypic variances were estimated according to the formula given by Johnson et al. [17]. Heritability in broad sense $\left(\mathrm{h}^{2}{ }_{\mathrm{b}}\right)$ was estimated according to the formula suggested by Johnson et al. [17] and Hanson [19]. Genotypic and phenotypic co-efficient of variations were estimated according to Burton [20] and Singh et al. [21]. Genetic advance was calculated following formula given by Johnson et al. [17] and Allard [22]. Genetic advance in percent of mean was calculated by the formula of Comstock et al. [23]. The phenotypic correlations were estimated by the formula suggested by Miller et al. [24]. Correlation coefficient were further partitioned into components of direct and indirect effects by path coefficient analysis originally developed by Wright [25] and later described by Dewey and Lu [26].

\section{Result and Discussion}

The analysis of variance of different tomato genotypes for yield and yield contributing traits are shown in Table 1. Results showed a significant $(p<0.001)$ variation among the genotypes for different traits indicating presence of significant variability in the materials which can be exploited through selection. Importantly, the success of breeding 
program depends upon quantum of variability present in the available germplasm. Significant variability among the genotypes for various characters except number of primary branches per plant was also reported [27]. The highest plant height $(149.17 \mathrm{~cm})$ was observed in BARI Tomato- 11 which was statistically significant and different from all other genotypes. The lowest value $(58.13 \mathrm{~cm})$ was recorded in V1006422. The genotype V1006484 bears maximum number of primary branches (13.6) and the genotype Binatomato-10 bears minimum number of primary branches (5.93). The average numbers of primary branches were (9.42). C11 took maximum days (75.6) to flower and Binatomato-5 required the minimum number of days to first flowering (57.8). The average value of days to first flowering was (68). The genotype Binatomato-5 took minimum days (62) to $50 \%$ flowering and the genotype WP8 required the maximum number of days (88) to $50 \%$ flowering. Binatomato-10 bears maximum number (17.57) of fruit cluster and BARI Tomato-15 bears minimum number (3.54) of fruit cluster. The genotype Binatomato-3 was early maturing which took (90.33) days for first fruit maturity. The genotype Binatomato- 8 required highest number of days (128.67) for first fruit maturity. The genotype Bahar gave the highest mean value of individual fruit weight (217.91g) which was significantly superior to all other genotypes. The lowest fruit weight $(8.09 \mathrm{~g})$ was observed in V1045786. The genotype Bahar had highest $(10.50 \mathrm{~cm})$ fruit diameter on the other hand Binatomato-10 had lowest $(1.78 \mathrm{~cm})$ fruit diameter. The highest number of fruits (177.67) recorded in BARI Tomato-11 and the lowest number of fruits (13.67) was recorded in V1006136. Fruit harvesting duration was maximum (154.67) days for the genotype V1006484. Minimum harvesting duration (131.00) days were recorded for Binatomato-8. The genotype Bahar had highest (17.89\%) dry matter percentage. The lowest dry matter content was recorded for the genotype $\mathrm{C} 71$. Binatomato-10 had highest (7.10\%) TSS and the lowest (2.53\%) TSS content was recorded for V1045786. Highest AsA content was recorded in C71 (38.87mg \%). The genotype WP8 contain lowest amount $(16.13 \mathrm{mg} \%$ ) of AsA among the selected genotypes. The highest yield (6.43) $\mathrm{kg}$ per plant was obtained from
Binatomato- 9 and the lowest yielding $(0.37 \mathrm{~kg}$ per plant $)$ genotype was V1006136 (Table 2).

\subsection{Genetic Parameter Analysis}

A clear idea can be gained by comparing the relative amount of phenotypic coefficient of variation (PCV) and genotypic coefficient of variation (GCV) for the actual strength of variability. The PCV was higher than the GCV for plant height $(\mathrm{PCV}=543.48, \mathrm{GCV}=521.24)$; number of primary branches per plant $(\mathrm{PCV}=58.62, \mathrm{GCV}=44.48)$; days to first flowering ( $\mathrm{PCV}=87.99, \mathrm{GCV}=77.04)$; days to $50 \%$ flowering ( $\mathrm{PCV}=100.80, \mathrm{GCV}=88.93)$; days to first harvest ( $\mathrm{PCV}=96.22, \quad \mathrm{GCV}=93.62)$; individual fruit weight $(\mathrm{PCV}=2425.67, \quad \mathrm{GCV}=2418.64)$; fruits per plant $(\mathrm{PCV}=3566.48, \mathrm{GCV}=3554.12)$ and dry matter percentage ( $\mathrm{PCV}=62.34, \mathrm{GCV}=61.60)$ indicated the influence of environment for the expression of these characters (Table 3). Highest GCV (15.09\%) and PCV (22.19\%) for plant height in a study of 12 tomato genotypes received from AVRDC, Taiwan grown at BARI farm, Joydebpur [12]. High genotypic variance indicating more contribution of genetic component for the total variation. Mohamed et al. [28] reported GCV and PCV (16.41\%) and (17.91\%) respectively for number of primary branches for plant. Similar results were also found by Biswas and Mallik [29] and Kaushik et al. [30] for days to first flowering. Gadekar et al. [31] also reported maximum phenotypic (381.74) and genotypic (323.14) variance with highest GCV (42.18\%) for individual fruit weight in tomato. This indicated the possibility of obtaining higher selection response in respect of these traits. Bahar had highest and C71 had the lowest dry matter percentage. Dutta et al. [32] studied six tomato varieties including two advanced lines Bahar and E-6 and found dry matter content $4.85 \%, 4.70 \%$ and $4.50 \%$ for Bahar, Oxheart and Ratan, respectively, which is similar to the present findings. Prodan [33] found early and tall varieties had higher dry matter content than that of later and dwarf varieties which support the present results. 
Table 1. Analysis of variance (mean square) for different characters of 30 genotypes of tomato

\begin{tabular}{|c|c|cccccccccccccc}
\hline S.V & DF & PH & PB & DFF & $50 \%$ F & FC & FH & IFW & FD & FP & LH & DM & TSS & AsA & YP \\
\hline Rep. & 2 & 10.72 & 1.88 & 0.20 & 3.61 & 0.14 & 5.41 & 10.29 & 0.11 & 11.20 & 0.68 & 0.08 & 0.66 & 6.29 & 0.15 \\
Trt. & 29 & $1482.17^{* * *}$ & $13.91^{* * *}$ & $101.66^{* * *}$ & $142.18^{* * *}$ & $26.64^{* * *}$ & $257.32^{* * *}$ & $5175.41^{* * *}$ & $9.58^{* * *}$ & $4689.80^{* * *}$ & $119.40^{* * *}$ & $22.42^{* * *}$ & $3.25^{* * *}$ & $72.96^{* * *}$ & $5.12^{* * *}$ \\
Error & 58 & 20.79 & 1.33 & 4.60 & 6.06 & 0.05 & 2.35 & 5.01 & 0.05 & 5.43 & 0.23 & 0.09 & 0.24 & 2.40 & 0.09 \\
\hline
\end{tabular}

*** indicates significant at $p<0.001$

Here, Trt. = treatment, $\mathrm{PH}=$ Plant height; $\mathrm{PB}=$ Number of primary branches per plant; $\mathrm{DFF}=\mathrm{Days}$ to first flowering; $50 \% \mathrm{~F}=\mathrm{Days}$ to $50 \%$ flowering; $\mathrm{FC}=\mathrm{Number}$ of fruit cluster per plant; $\mathrm{FH}=\mathrm{Days}$ to firs harvest; IFW= Individual fruit weight; $F D=$ Fruit diameter; $F P=$ Fruits per plant; $\mathrm{YP}=$ Yield per plant; $\mathrm{LH}=$ Days to last harvest; $\mathrm{DM}=\mathrm{Dry}$ matter percentage; TSS= Total soluble solid; AsA = Ascorbic acid content.

Table 2. Average performance for 14 different traits (morphological and biochemical) of 30 genotypes of tomato

\begin{tabular}{|c|c|c|c|c|c|c|c|c|c|c|c|c|c|c|}
\hline Ge. & $\begin{array}{l}\mathrm{PH} \\
(\mathrm{cm})\end{array}$ & $\begin{array}{c}\text { PB } \\
\text { (no.) } \\
\end{array}$ & $\begin{array}{c}\text { DFF } \\
\text { (days) }\end{array}$ & $\begin{array}{l}50 \% \mathrm{~F} \\
\text { (days) }\end{array}$ & $\begin{array}{c}\mathrm{FC} \\
\text { (no.) }\end{array}$ & $\begin{array}{c}\mathrm{FH} \\
\text { (days) }\end{array}$ & $\begin{array}{c}\text { IFW } \\
(\mathrm{g})\end{array}$ & $\begin{array}{l}\text { FD } \\
(\mathrm{cm})\end{array}$ & $\begin{array}{c}\text { FP } \\
\text { (no.) }\end{array}$ & $\begin{array}{c}\text { YP } \\
(\mathrm{kg})\end{array}$ & $\begin{array}{c}\text { LH } \\
\text { (days) }\end{array}$ & $\begin{array}{l}\mathrm{DM} \\
(\%)\end{array}$ & $\begin{array}{l}\text { TSS } \\
(\%)\end{array}$ & $\begin{array}{c}\begin{array}{c}\text { AsA } \\
(\mathrm{mg} / 100 \mathrm{~g})\end{array} \\
\end{array}$ \\
\hline 1 & $74 \mathrm{mn}$ & $11.07 \mathrm{bcd}$ & $61 \mathrm{gh}$ & 71jkl & $5.50 \mathrm{kl}$ & $110 \mathrm{jk}$ & $83.96 \mathrm{~g}$ & $7.08 \mathrm{de}$ & $29.67 \mathrm{jkl}$ & $2.42 \mathrm{gh}$ & 1360 & $10.951 \mathrm{~m}$ & 3.63hij & $24.93 \mathrm{efg}$ \\
\hline 2 & 92.8hij & $12.20 \mathrm{abc}$ & $65 \mathrm{f}$ & $82 \mathrm{bcde}$ & $4.60 \mathrm{~m}$ & $119 \mathrm{gh}$ & $32.49 \mathrm{r}$ & $5.06 \mathrm{kl}$ & $13.67 \mathrm{r}$ & $0.37 \mathrm{p}$ & $152 \mathrm{c}$ & $8.00 \mathrm{~s}$ & $4.57 \mathrm{cdefg}$ & $16.87 \mathrm{~lm}$ \\
\hline 3 & $58.13 q$ & $13.07 \mathrm{a}$ & 60hi & 71jlk & $7.60 \mathrm{f}$ & $120 \mathrm{~g}$ & $45.27 \mathrm{q}$ & $6.76 \mathrm{ef}$ & $15.00 \mathrm{qr}$ & 0.73nop & $154 b$ & 12.26hi & $4.53 \mathrm{cdefg}$ & 22.73ghi \\
\hline 4 & 71.53 no & $13.00 \mathrm{a}$ & $69 \mathrm{de}$ & $81 \mathrm{cdef}$ & 6.74ghi & $120 \mathrm{fg}$ & $24.29 \mathrm{~s}$ & $6.93 \mathrm{de}$ & $20.67 \mathrm{op}$ & 0.61 op & $150 \mathrm{e}$ & $9.58 \mathrm{qr}$ & 3.67hij & $20.53 \mathrm{ijk}$ \\
\hline 5 & $124.07 \mathrm{c}$ & $13.60 \mathrm{a}$ & 61ghi & 681 & $6.50 \mathrm{hij}$ & $122 \mathrm{def}$ & $45.31 q p$ & $7.05 \mathrm{de}$ & 18.33 opq & 0.89 no & $155 \mathrm{a}$ & $14.31 \mathrm{e}$ & 3.50hijk & $21.27 \mathrm{hij}$ \\
\hline 6 & 69.33nop & $12.27 \mathrm{abc}$ & $58 \mathrm{hi}$ & $70 \mathrm{kl}$ & $5.60 \mathrm{k}$ & $108 \mathrm{k}$ & $8.09 \mathrm{t}$ & $2.82 q$ & $127.00 \mathrm{c}$ & $0.98 \mathrm{nom}$ & $151 d$ & $14.94 \mathrm{~d}$ & 2.531 & $27.13 \mathrm{cde}$ \\
\hline 7 & $65.87 \mathrm{op}$ & 7.33ghi & $72 \mathrm{bcd}$ & $84 a b c$ & $4.60 \mathrm{~m}$ & $124 \mathrm{~cd}$ & 53.390 & $4.861 \mathrm{~m}$ & $17.00 \mathrm{pqr}$ & 0.92 nom & $141 \mathrm{k}$ & $8.43 \mathrm{~s}$ & $4.70 \mathrm{~cd}$ & $25.67 \mathrm{def}$ \\
\hline 8 & $112.8 \mathrm{de}$ & $9.13 \mathrm{efg}$ & $67 \mathrm{ef}$ & 74ijk & $6.23 \mathrm{j}$ & $123 \mathrm{~cd}$ & $58.54 n$ & $6.38 \mathrm{gh}$ & $35.67 \mathrm{~h}$ & $1.76 \mathrm{jk}$ & $138 \mathrm{n}$ & $11.29 \mathrm{kl}$ & $5.57 \mathrm{~b}$ & 22.00hi \\
\hline 9 & 91.93hijk & $8.40 \mathrm{fgh}$ & $74 a b$ & $88 \mathrm{a}$ & $6.87 \mathrm{~g}$ & $122 \mathrm{def}$ & 75.88ij & $4.64 \mathrm{mn}$ & 29.33jkl & $2.12 \mathrm{hij}$ & $150 \mathrm{e}$ & $8.27 \mathrm{~s}$ & 3.03ijkl & $16.13 \mathrm{~m}$ \\
\hline 10 & $84.53 \mathrm{kl}$ & 12.93ab & $59 \mathrm{hi}$ & 681 & $7.33 \mathrm{f}$ & $111 \mathrm{jk}$ & $104.52 d$ & $6.54 \mathrm{fg}$ & $34.33 \mathrm{hi}$ & $3.10 \mathrm{ef}$ & 140kl & $10.32 \mathrm{nop}$ & $3.77 \mathrm{ghi}$ & $23.47 \mathrm{fgh}$ \\
\hline 11 & 72.6no & $8.60 \mathrm{efg}$ & $76 \mathrm{a}$ & 83 bcde & 5.241 & $119 \mathrm{gh}$ & $69.73 \mathrm{kl}$ & 4.45 no & $30.67 \mathrm{ij}$ & $1.74 \mathrm{jk}$ & $1391 \mathrm{~m}$ & $9.37 \mathrm{r}$ & 3.63hij & $29.33 b$ \\
\hline 12 & $80.41 \mathrm{~m}$ & $8.13 \mathrm{fgh}$ & $72 \mathrm{bcd}$ & $85 \mathrm{ab}$ & $8.43 \mathrm{e}$ & $123 \mathrm{de}$ & $43.21 \mathrm{q}$ & $4.831 \mathrm{~m}$ & $30.33 \mathrm{jk}$ & $1.16 \mathrm{lmn}$ & 144hi & 9.99opq & $2.93 \mathrm{jkl}$ & $24.93 \mathrm{efg}$ \\
\hline 13 & 88.67ijk & $11.87 \mathrm{abc}$ & 61ghi & $71 \mathrm{jkl}$ & $9.87 \mathrm{c}$ & 117hi & 77.58ih & $7.14 d$ & $43.33 \mathrm{fg}$ & $3.25 \mathrm{ef}$ & $142 \mathrm{j}$ & $10.80 \mathrm{mn}$ & $4.63 \mathrm{cdef}$ & $27.87 \mathrm{bcd}$ \\
\hline 14 & $132.8 \mathrm{~b}$ & $9.53 \mathrm{def}$ & $72 \mathrm{bcd}$ & $83 \mathrm{bcde}$ & $6.47 \mathrm{hij}$ & 119ghi & $66.36 \mathrm{ml}$ & $4.57 \mathrm{mn}$ & $30.67 \mathrm{ij}$ & $1.87 \mathrm{ijk}$ & $152 \mathrm{c}$ & $7.96 \mathrm{~s}$ & $5.20 \mathrm{bc}$ & $38.87 \mathrm{a}$ \\
\hline
\end{tabular}




\begin{tabular}{|c|c|c|c|c|c|c|c|c|c|c|c|c|c|c|}
\hline Ge. & $\begin{array}{l}\mathrm{PH} \\
(\mathrm{cm})\end{array}$ & $\begin{array}{l}\text { PB } \\
\text { (no.) }\end{array}$ & $\begin{array}{l}\text { DFF } \\
\text { (days) }\end{array}$ & $\begin{array}{l}50 \% \mathrm{~F} \\
\text { (days) }\end{array}$ & $\begin{array}{l}\mathrm{FC} \\
\text { (no.) }\end{array}$ & $\begin{array}{c}\mathrm{FH} \\
\text { (days) }\end{array}$ & $\begin{array}{l}\text { IFW } \\
(\mathrm{g})\end{array}$ & $\begin{array}{l}\mathrm{FD} \\
(\mathrm{cm})\end{array}$ & $\begin{array}{l}\text { FP } \\
\text { (no.) }\end{array}$ & $\begin{array}{c}\text { YP } \\
(\mathrm{kg})\end{array}$ & $\begin{array}{l}\mathrm{LH} \\
\text { (days) }\end{array}$ & $\begin{array}{l}\mathrm{DM} \\
(\%)\end{array}$ & $\begin{array}{l}\text { TSS } \\
(\%)\end{array}$ & $\begin{array}{c}\mathrm{AsA} \\
(\mathrm{mg} / 100 \mathrm{~g})\end{array}$ \\
\hline 15 & $75.73 \mathrm{mn}$ & $9.33 \mathrm{def}$ & $74 \mathrm{ab}$ & 84abcd & $6.73 \mathrm{ghi}$ & $128 \mathrm{ab}$ & $71.10 \mathrm{k}$ & $4.38 \mathrm{no}$ & $26.67 \mathrm{klm}$ & $1.79 \mathrm{jk}$ & $140 \mathrm{k}$ & $16.33 \mathrm{c}$ & 3.47hijk & $17.601 \mathrm{~m}$ \\
\hline 16 & $149.17 \mathrm{a}$ & $6.20 \mathrm{i}$ & $70 \mathrm{cde}$ & 78 fghi & $5.27 \mathrm{kl}$ & $113 \mathrm{j}$ & $8.26 \mathrm{t}$ & $2.70 \mathrm{q}$ & $177.67 \mathrm{a}$ & $1.40 \mathrm{klm}$ & 1370 & $10.40 \mathrm{on}$ & $4.83 \mathrm{bc}$ & $24.93 \mathrm{efg}$ \\
\hline 17 & $118.87 \mathrm{~cd}$ & $8.27 \mathrm{fgh}$ & $73 a b c$ & $86 a b$ & $5.45 \mathrm{kl}$ & $123 \mathrm{de}$ & $98.3 \mathrm{e}$ & $5.47 \mathrm{jI}$ & $26.331 \mathrm{~m}$ & $2.55 \mathrm{gh}$ & $152 \mathrm{c}$ & $16.03 \mathrm{c}$ & 3.43hijk & $27.13 \mathrm{bcde}$ \\
\hline 18 & $100.27 \mathrm{fg}$ & $8.33 \mathrm{fgh}$ & $73 \mathrm{ab}$ & $83 \mathrm{bcde}$ & $3.54 \mathrm{n}$ & 119ghi & $63.07 \mathrm{~m}$ & $7.13 \mathrm{~d}$ & $40.67 \mathrm{~g}$ & $2.55 \mathrm{gh}$ & $149 \mathrm{f}$ & $14.77 \mathrm{de}$ & 3.63hij & $18.33 \mathrm{klm}$ \\
\hline 19 & $63.8 \mathrm{pq}$ & $8.13 \mathrm{fg}$ & $74 a b$ & $80 \mathrm{cdef}$ & $7.63 \mathrm{f}$ & 1031 & $80.24 \mathrm{~h}$ & $6.24 \mathrm{gh}$ & $35.00 \mathrm{~h}$ & $2.75 \mathrm{fg}$ & $144 \mathrm{~h}$ & $11.99 \mathrm{ij}$ & 3.63hij & $18.33 \mathrm{klm}$ \\
\hline 20 & 89.33ijk & $8.20 \mathrm{fgh}$ & $70 \mathrm{cde}$ & 79efgh & $4.60 \mathrm{~m}$ & 1051 & 67.161 & $6.12 \mathrm{~h}$ & $25.00 \mathrm{mn}$ & $1.48 \mathrm{kl}$ & $142 \mathrm{j}$ & $12.56 \mathrm{gh}$ & $4.67 \mathrm{cde}$ & $18.33 \mathrm{klm}$ \\
\hline 21 & $109.13 \mathrm{e}$ & $8.40 \mathrm{fgh}$ & $74 \mathrm{ab}$ & $86 \mathrm{ba}$ & $3.77 \mathrm{n}$ & $108 \mathrm{k}$ & $101.41 \mathrm{ed}$ & $8.07 \mathrm{~b}$ & $22.00 \mathrm{no}$ & $2.20 \mathrm{hij}$ & $153 \mathrm{bc}$ & $11.84 \mathrm{ij}$ & $4.57 \mathrm{cdefg}$ & $20.53 \mathrm{ijk}$ \\
\hline 22 & 90.8hijk & $8.67 \mathrm{efg}$ & $73 a b c$ & $83 \mathrm{bcde}$ & $6.40 \mathrm{ij}$ & $124 \mathrm{~cd}$ & $217.91 \mathrm{a}$ & $10.50 \mathrm{a}$ & $20.33 \mathrm{op}$ & $4.12 \mathrm{bc}$ & $143 \mathrm{i}$ & $17.89 \mathrm{a}$ & 3.53hijk & $19.07 \mathrm{jkl}$ \\
\hline 23 & $85.07 \mathrm{kl}$ & $10.40 \mathrm{cde}$ & $65 \mathrm{fg}$ & 681 & $12.60 \mathrm{~b}$ & $95 \mathrm{~m}$ & $48.97 \mathrm{p}$ & $4.32 \mathrm{no}$ & $45.67 \mathrm{f}$ & $2.17 \mathrm{hij}$ & $141 \mathrm{k}$ & $12.49 \mathrm{gh}$ & 3.83fghi & $16.87 \mathrm{~lm}$ \\
\hline 24 & $87.2 \mathrm{jkl}$ & $8.20 \mathrm{fgh}$ & 72abcd & 80defg & $4.76 \mathrm{~m}$ & $123 \mathrm{de}$ & $138.76 b$ & $7.65 \mathrm{c}$ & 21.33 no & $2.79 \mathrm{fg}$ & $143 \mathrm{i}$ & $11.66 \mathrm{jk}$ & 3.53hijk & $20.53 \mathrm{ijk}$ \\
\hline 25 & 88.93ijk & $8.20 \mathrm{fgh}$ & $61 \mathrm{gh}$ & 701 & $6.80 \mathrm{gh}$ & $90 n$ & $94.49 \mathrm{f}$ & $5.16 \mathrm{jkl}$ & $26.67 \mathrm{klm}$ & 2.30ghi & $139 \mathrm{mn}$ & $16.90 \mathrm{~b}$ & $2.77 \mathrm{kl}$ & $19.07 \mathrm{ijk}$ \\
\hline 26 & 90.93hijk & 7.73fghi & $71 \mathrm{bcd}$ & $75 \mathrm{ij}$ & $5.57 \mathrm{kl}$ & $125 \mathrm{bc}$ & $83.93 \mathrm{~g}$ & $3.90 \mathrm{p}$ & $47.00 \mathrm{f}$ & $3.83 \mathrm{~cd}$ & 1370 & $12.98 \mathrm{~g}$ & 3.03ijkl & $28.60 \mathrm{bc}$ \\
\hline 27 & 107.07ef & $8.47 \mathrm{fgh}$ & $58 \mathrm{i}$ & $62 \mathrm{~m}$ & $12.60 \mathrm{~b}$ & $116 \mathrm{i}$ & $73.27 \mathrm{kj}$ & $4.15 \mathrm{po}$ & $64.67 \mathrm{~d}$ & $4.41 \mathrm{~b}$ & $147 \mathrm{~g}$ & $14.81 \mathrm{~d}$ & $6.53 a$ & $25.67 \mathrm{def}$ \\
\hline 28 & $97.93 \mathrm{gh}$ & $10.47 \mathrm{cde}$ & $69 \mathrm{cde}$ & 75hi & $9.53 \mathrm{dc}$ & $129 a$ & 56.83 on & $5.24 \mathrm{jk}$ & $55.67 \mathrm{e}$ & $3.06 \mathrm{ef}$ & $131 \mathrm{p}$ & $11.75 \mathrm{jk}$ & 4.00defgh & $27.13 \mathrm{bcde}$ \\
\hline 29 & $95.27 \mathrm{ghi}$ & $6.67 \mathrm{hi}$ & $74 \mathrm{ab}$ & 76ghi & $9.20 \mathrm{~d}$ & $121 \mathrm{efg}$ & $123.20 \mathrm{c}$ & $5.62 \mathrm{i}$ & $53.33 \mathrm{e}$ & $6.43 a$ & 144hi & $13.80 \mathrm{f}$ & $3.87 \mathrm{efgh}$ & $26.40 \mathrm{cde}$ \\
\hline 30 & $134.73 b$ & $5.93 \mathrm{i}$ & $61 \mathrm{hi}$ & 691 & $17.57 \mathrm{a}$ & $124 \mathrm{~cd}$ & $22.15 \mathrm{~s}$ & $1.78 \mathrm{r}$ & $154.33 b$ & $3.36 \mathrm{de}$ & 138 on & $9.87 \mathrm{pq}$ & 7.10a & 22.73ghi \\
\hline Min. & 58.13 & 5.93 & 57.8 & 62.33 & 3.54 & 90.33 & 8.09 & 1.78 & 13.67 & 0.37 & 131.00 & 7.96 & 2.53 & 16.13 \\
\hline Max. & 149.17 & 13.60 & 75.6 & 87.67 & 17.57 & 128.67 & 217.91 & 10.50 & 177.67 & 6.43 & 154.67 & 17.89 & 7.10 & 38.87 \\
\hline Avr. & 93.46 & 9.42 & 67.99 & 77.02 & 7.12 & 116.77 & 71.26 & 5.58 & 43.93 & 2.30 & 144.18 & 12.08 & 4.08 & 23.10 \\
\hline SE & 4.06 & 0.39 & 1.06 & 1.26 & 0.54 & 1.69 & 7.58 & 0.33 & 7.22 & 0.24 & 1.15 & 0.50 & 0.19 & 0.90 \\
\hline SD & 22.23 & 2.15 & 5.82 & 6.88 & 2.98 & 9.26 & 41.53 & 1.79 & 39.54 & 1.31 & 6.31 & 2.73 & 1.04 & 4.93 \\
\hline $\mathrm{LSD}_{0.05}$ & 7.45 & 1.89 & 3.50 & 4.02 & 0.34 & 2.50 & 3.65 & 0.35 & 3.80 & 0.49 & 0.78 & 0.48 & 0.80 & 2.53 \\
\hline
\end{tabular}

Here, Ge 1=V1007282, 2=V1006136, 3=V1006422, 4=V1006186, 5=V1006484, 6=V1045786, 7= V1063607, 8=WP7, 9=WP8, 10=WP10, 11=C11, 12=C41, 13=C51, 14=C71, 15= BARI Tomato-9, $16=$ BARI Tomato-1 1, 17= BARI Tomato-14, 18= BARI Tomato-15, 19= BARI Tomato-8, 20= Phili-1, 21= Phili-2, 22= Bahar, 23= Binatomato-2, $24=$ Binatomato-7, $25=$ Binatomato-3, 26= Binatomato-4, $27=$ Binatomato-5, 28= Binatomato-8, $29=$ Binatomato-9, 30= Binatomato-10. $\mathrm{Ge}=$ Genotype, $\mathrm{PH}=$ Plant height; $\mathrm{PB}=$ Number of primary branches per plant; $\mathrm{DFF}=$ Days to first flowering; $50 \% \mathrm{~F}=\mathrm{Days}$ to $50 \%$ flowering; $\mathrm{FC}=\mathrm{Number}$ of fruit cluster per plant; $\mathrm{FH}=\mathrm{Days}$ to first harvest; IFW= Individual fruit weight; FD= Fruit diameter; FP= Fruits per plant; YP= Yield per plant; LH= Days to last harvest; DM= Dry matter percentage; TSS= Total soluble solid; AsA = Ascorbic acid content, Min.= Minimum, Max.= Maximum, Avr.= Average, Sd. Er.= Standard Error, Sd. Dev.= Standard deviation, LSD $0.05=$ Least significant difference at $5 \%$ level of significance 
Table 3. Genetic Parameters of different characters of 30 tomato genotypes

\begin{tabular}{|c|c|c|c|c|c|c|}
\hline Traits & $\begin{array}{l}\text { Genotypic } \\
\text { variance }\end{array}$ & $\begin{array}{c}\text { Phenotypic } \\
\text { variance }\end{array}$ & $\begin{array}{c}\text { Heritability } \\
(\%)\end{array}$ & $\begin{array}{c}\text { GCV } \\
(\%)\end{array}$ & $\begin{array}{c}\text { PCV } \\
(\%)\end{array}$ & $\begin{array}{l}\text { GA } \\
(\%)\end{array}$ \\
\hline PH & 487.13 & 507.92 & 95.91 & 521.24 & 543.48 & 44.53 \\
\hline PB & 4.19 & 5.52 & 75.87 & 44.48 & 58.62 & 3.67 \\
\hline DFF & 32.35 & 36.95 & 87.56 & 77.04 & 87.99 & 10.96 \\
\hline $50 \% \mathrm{~F}$ & 45.37 & 51.43 & 88.22 & 88.93 & 100.80 & 13.03 \\
\hline $\mathrm{FC}$ & 8.87 & 8.91 & 99.49 & 124.51 & 125.15 & 6.12 \\
\hline FH & 84.99 & 87.34 & 97.31 & 93.62 & 96.22 & 18.73 \\
\hline IFW & 1723.47 & 1728.48 & 99.71 & 2418.64 & 2425.67 & 85.40 \\
\hline FD & 3.18 & 3.22 & 98.57 & 56.89 & 57.72 & 3.65 \\
\hline FP & 1561.46 & 1566.89 & 99.65 & 3554.12 & 3566.48 & 81.26 \\
\hline YP & 1.68 & 1.77 & 94.84 & 72.82 & 76.78 & 2.60 \\
\hline LH & 39.72 & 39.95 & 99.43 & 33.61 & 33.81 & 12.95 \\
\hline $\mathrm{DM}$ & 7.44 & 7.53 & 98.83 & 61.60 & 62.34 & 5.59 \\
\hline TSS & 1.00 & 1.25 & 80.51 & 24.59 & 30.54 & 1.85 \\
\hline AsA & 23.52 & 25.92 & 90.75 & 101.83 & 112.21 & 9.52 \\
\hline
\end{tabular}

Here, $\mathrm{PH}=$ Plant height; $\mathrm{PB} .=$ Number of primary branches per plant; $\mathrm{DFF}=$ Days to first flowering; $50 \% \mathrm{~F}=$ Days to $50 \%$ flowering; $\mathrm{FC}=\mathrm{Number}$ of fruit cluster per plant; $\mathrm{FH}=$ Days to first harvest; IFW= Individual fruit weight; $\mathrm{FD}=$ Fruit diameter; $\mathrm{FP}=$ Fruits per plant; $\mathrm{YP}=\mathrm{Yield}$ per plant; $\mathrm{LH}=\mathrm{Days}$ to last harvest; $\mathrm{DM}=$ Dry matter percentage; TSS= Total soluble solid; AsA = Ascorbic acid content; $\mathrm{GCV}=$ Genotypic co-efficient of variation; $\mathrm{PCV}=$ Phenotypic co-efficient of variation; $\mathrm{GA}=$ Genetic advance; $\mathrm{GA}(\%)=$ Genetic advance in percentage of mean.

Determinate plants had a lower content of dry matter than the indeterminate [34], mainly as a result of reduced leaf surface area. Indeterminate plant types were best for use in breeding for a high content of dry matter [35]. Number of fruit cluster per plant $(\mathrm{PCV}=125.15, \mathrm{GCV}=124.51)$; fruit diameter $(\mathrm{PCV}=57.72, \mathrm{GCV}=56.89)$ and days to last harvest $(\mathrm{PCV}=33.81, \mathrm{GCV}=33.61)$ showed relatively low difference between PCV and GCV. This indicates the low impact of environment on the expression of characters and hence, they could be improved by following different phenotypic selections like directional, disruptive and stabilized selections. Tasisa et al. [36] obtained slightly higher PCV $(12.98 \%)$ than GCV (12.46) for number of fruit cluster per plant. Chernet et al. [37] recorded radial and polar diameter of fruit and recorded higher PCV $(10.09 \& 13.95)$ than GCV (10.04 \& 13.90) for dry matter percentage. Similar result was also found by Osekita and Ademiluyi [38] for days to last harvest. Vinod et al. [39] reported PCV (8.14) and GCV (7.29) for TSS content. High values of GCV are an indication of high genetic variability among the germplasm and thus the scope for improvement of these characters through simple selection would be better. The differences between PCV and GCV was minimum for number of fruit cluster per plant, fruit diameter, days to last harvest and dry matter percentage suggesting that these traits were least affected by environment.

\section{Heritability and Genetic advance}

By GCV and PCV alone, it is not possible to determine the amount of variation which is heritable. The heritability along with genetic advance is more meaningful and helps in predicating the resultant effect of selection on phenotypic expression [17]. High heritability with high genetic advance showed in plant height $\left(\mathrm{h}_{\mathrm{b}}^{2}=95.91, \mathrm{GA}=44.53\right)$; individual fruit weight $\left(\mathrm{h}_{\mathrm{b}}^{2}=99.71, \mathrm{GA}=85.40\right)$; fruits per plant $\left(\mathrm{h}_{\mathrm{b}}^{2}\right.$ $=99.5, \mathrm{GA}=81.26$ ) indicating that these characters were under additive genetic effects and that this trait could be considered as reliable indices for selection (Table 3). Similar results were also reported by other researchers in tomato. Many researchers found high genetic advance for individual fruit weight [40-42] and plant height [43-45]. For number of fruits per plant Mehta and Asati [46] observed moderate heritability (54.70) with genetic advance in percentage of mean (27.86). Tasis et al. [47] reported high heritability $(99 \%)$ with genetic advance (11.32) and genetic advance in percentage of mean (32.54\%). Meitei et al. [27] observed high values of heritability (98.55) associated with high genetic advance (45.91) and high genetic advance in percentage of mean (132.30\%) for number of fruits per plant. High heritability with low genetic advance observed in number of primary branches $\left(\mathrm{h}_{\mathrm{b}}^{2}=75.87, \mathrm{GA}=3.67\right)$; days to first flowering $\left(\mathrm{h}_{\mathrm{b}}{ }_{\mathrm{b}}=87.56, \mathrm{GA}=10.96\right)$; days to $50 \%$ flowering $\left(\mathrm{h}_{\mathrm{b}}^{2}=88.22, \mathrm{GA}=13.03\right)$; number of fruit cluster per plant $\left(\mathrm{h}_{\mathrm{b}}^{2}=99.49, \mathrm{GA}=6.12\right)$; days to first harvest $\left(\mathrm{h}_{\mathrm{b}}{ }_{\mathrm{b}}=97.31, \quad \mathrm{GA}=18.73\right)$; fruit diameter $\left(\mathrm{h}_{\mathrm{b}}^{2}=98.57\right.$, $\mathrm{GA}=3.65)$; yield per plant $\left(\mathrm{h}_{\mathrm{b}}{ }_{\mathrm{b}}=94.84, \mathrm{GA}=2.6\right)$; days to last harvest $\left(\mathrm{h}_{\mathrm{b}}^{2}=99.43, \mathrm{GA}=12.95\right)$; dry matter percentage $\left(\mathrm{h}_{\mathrm{b}}^{2}\right.$ $=98.83, \mathrm{GA}=5.59)$; total soluble solid $\left(\mathrm{h}_{\mathrm{b}}^{2}=80.51, \mathrm{GA}=1.85\right)$; AsA content $\left(\mathrm{h}_{\mathrm{b}}{ }_{\mathrm{b}}=90.75, \mathrm{GA}=9.52\right)$. This situation indicated limited scope for the improvement of this character through selection but hybridization followed by progeny selection will be effective. Kaushik et al. [30] also reported high amount of heritability $(69.20 \%)$ but low genetic advance as percentage of mean $(3.2 \%)$ for days to first flowering. Mohanty [48] reported that the range of days to $50 \%$ flowering was 51 to 54 days and low heritability and low 
genetic advance as percentage of mean. Meena and Bahadur [45] found high heritability (100) with low genetic advance (16.85) and low genetic advance as percentage of mean $(27.40 \%)$ for days to $50 \%$ flowering. Similar results were also found by Tasisa et al. [36] and Meitei et al. [27] for number of fruit cluster per plant. Many researchers reported high heritability with low genetic advance for days to maturity [49-51]. Osekita and Ademiluyi [38] found similar findings for days to last harvest. Meena and Bahadur [45] found high heritability (98\%) and low genetic advance (2.16) and genetic advance in percentage of mean $(51.59 \%)$ for TSS content. Mohamed et al. [28] also reported high heritability (80\%) with low genetic advance (3.47) for AsA content. From the present study it is found that smaller fruit have more AsA than the larger one. Bahar had the highest fruit diameter $(10.5 \mathrm{~cm})$ and fruit weight $(217.90 \mathrm{~g})$ but the AsA content is very low (19.06 mg\%) comparing other small sized fruit. Barooah and Mohan [52] studied 11 varieties and found that Sioux having largest fruit size had less AsA content and Chickugrande, the smallest fruit had highest ascorbic content $(26.30 \mathrm{mg} \%)$, which were in agreement with this research findings. The better indicators of heritable proportion of variation estimation are heritability estimation. The high heritability indicates the effectiveness of selection based on phenotypic traits but does not necessarily mean a high genetic gain for a particular trait. So, consideration of both, heritability and genetic advance is more important for predicting effective selection than heritability alone. Johnson et al. [17] reported that heritability estimates along with genetic advance would be more rewarding than heritability alone in predicting the consequential effect of selection to choose the best individual. High values of heritability with high genetic advance were observed for individual fruit weight followed by fruits per plant and plant height indicted that these traits were under the control of addictive gene action and directional selection for these characters in the genetically diverse material could be effective for desired genetic improvement. Similar findings were reported earlier by Mehta and Asati [46], Mohanty [48] in tomato.

\subsection{Correlation Co-efficient Studies}

A crop breeding program aimed at increasing the yield requires consideration not only of yield but also of its associate components that have direct or indirect impact on yield. Correlation analyses give an insight into the genetic variability present in populations. Correlation coefficient analysis measures the mutual relationship between various plant characters and determines the component characters on which selection can be based for improvement in yield. Correlation studies were made among 14 characters of which yield per plant were positively correlated with individual fruit weight (0.609) and number of fruit cluster per plant (0.396) (Table 4). The significant and positive association between the traits suggested additive genetic model thereby less affected by the environmental fluctuation. Similar results have also been reported by Susic et al. [53] and Rani et al. [54] for individual fruit weight. Reddy et al. [55] also observed positive association between number of fruit cluster and fruit yield per plant. Similar findings were also reported by Kumar and Dudi [56] for fruit cluster. Yield per plant also showed positive but insignificant relationship with plant height (0.159), fruit diameter (0.105), number of fruits per plant (0.126), dry matter (\%) (0.352), TSS $(0.126)$ and AsA (0.163). Sharma and Singh [57] reported positive insignificant association with plant height, number of fruits per plant and TSS. Similar findings were also reported by Golani et al. [44] and Manna and Paul [34].

But Islam et al. [58] observed positive significant association between yield and fruit diameter, which is contradictory with the present research finding. On the other hand de Souza et al. [59] reported positive insignificant association with fruit diameter, which support the present results. Similar results also reported by Anitha et al. [60] for AsA. The positive and non-significant association referred information of inherent relation among the pairs of combination. Yield per plant showed negative significant relationship with number of primary branch per plant (-0.421) but previously it was reported by many researchers like Reddy et al. [55] and Sharma and Singh [57] that yield was positively significant with number of primary branches per plant, which are not in agreement with our findings. But Meena and Bahadur [61] reported negative significant association between yield and number of primary branches, which approves reports of the present investigation. Negative but insignificant association was found with days to $50 \%$ flowering $(-0.240)$, days to last harvest $(-0.318)$. The negative and non-significant association referred a complex linked of relation among the pair of combinations.

Character association revealed the mutual relationship between two characters, and it is important parameters for taking a decision regarding the nature of selection to be followed for improvement in the crop under study. The overall results indicated that yield per plant was positively associated with days to first flowering (0.099), number of fruit cluster per plant(0.396), individual fruit weight (0.609), number of fruits per plant $(0.126)$ and negatively associated with number of primary branches per plant (-0.421) and days to last harvest (-0.318). The traits which do not show any significant association or very negligible amount of association can be discarded to reduce the number of traits to characterize. This correlation result can be used as basis for character discard if similar research is conducted in the future using additional morphological traits. Elimination of redundant traits will reduce the workload of researcher and will render characterization less cumbersome and more efficient. 
Genetic Variability and Traits Association Analysis of Tomato (Lycopersicon esculentum L.)

Genotypes for Yield and Quality Attributes

Table 4. Phenotypic correlation co-efficients between yield and yield contributing traits

\begin{tabular}{|c|c|c|c|c|c|c|c|c|c|c|c|c|c|c|}
\hline & YP & $\mathrm{PH}$ & PB & DFF & $50 \% \mathrm{~F}$ & FC & $\mathrm{FH}$ & IFW & $\mathrm{FD}$ & FP & $\mathrm{LH}$ & DM & TSS & AsA \\
\hline YP & 1.00 & 0.159 & $-0.421 *$ & 0.099 & -0.240 & $0.396^{*}$ & 0.078 & $0.609 * * *$ & 0.105 & 0.126 & -0.318 & 0.352 & 0.126 & 0.163 \\
\hline PH & & 1.000 & -0.336 & 0.005 & -0.047 & 0.184 & 0.146 & -0.105 & -0.239 & $0.476^{* *}$ & 0.036 & -0.022 & $0.488^{* *}$ & 0.239 \\
\hline PB & & & 1.000 & $-0.546^{* *}$ & -0.331 & -0.100 & -0.054 & -0.209 & 0.300 & -0.326 & $0.362 *$ & -0.062 & -0.221 & -0.040 \\
\hline DFF & & & & 1.000 & $0.864 * * *$ & $-0.422^{*}$ & 0.312 & 0.322 & 0.157 & -0.255 & -0.027 & -0.086 & -0.259 & -0.022 \\
\hline $50 \% \mathrm{~F}$ & & & & & 1.000 & $-0.554 * * *$ & 0.335 & 0.178 & 0.181 & -0.322 & 0.222 & -0.217 & -0.271 & -0.088 \\
\hline FC & & & & & & 1.000 & -0.008 & -0.163 & $-0.388^{*}$ & $0.406^{*}$ & -0.245 & -0.021 & $0.499^{*}$ & 0.047 \\
\hline $\mathrm{FH}$ & & & & & & & 1.000 & 0.053 & 0.030 & -0.058 & 0.025 & -0.159 & 0.152 & 0.254 \\
\hline IFW & & & & & & & & 1.000 & $0.671 * * *$ & $-0.442 *$ & -0.081 & $0.431 *$ & -0.223 & -0.093 \\
\hline FD & & & & & & & & & 1.000 & $-0.653 * * *$ & 0.211 & 0.245 & -0.222 & -0.280 \\
\hline FP & & & & & & & & & & 1.000 & -0.276 & -0.029 & 0.336 & 0.199 \\
\hline LH & & & & & & & & & & & 1.000 & 0.034 & -0.065 & -0.065 \\
\hline $\mathrm{DM}$ & & & & & & & & & & & & 1.000 & -0.300 & -0.228 \\
\hline TSS & & & & & & & & & & & & & 1.000 & 0.172 \\
\hline AsA & & & & & & & & & & & & & & 1.000 \\
\hline
\end{tabular}

$*, * *$ and $* * * \quad$ indicates significant at $\mathrm{p}<0.05, \mathrm{p}<0.01$ and $\mathrm{p}<0.001$

Here,

$\mathrm{PH}=$ Plant height; $\mathrm{PB}=$ Number of primary branches per plant; $\mathrm{DFF}=$ Days to first flowering; $50 \% \mathrm{~F}=$ Days to $50 \%$ flowering; $\mathrm{FC}=$ Number of fruit cluster per plant; $\mathrm{FH}=\mathrm{Days}$ to first harvest;

$\mathrm{IFW}=$ Individual fruit weight; FD= Fruit diameter; FP= Fruits per plant; YP= Yield per plant; LH= Days to last harvest; DM= Dry matter percentage; TSS= Total soluble solid; AsA = Ascorbic acid content. 


\subsection{Path Co-efficient Studies}

Yield is the sum total of the many component characters which directly or indirectly contributed to it. Correlation studies give an idea about the positive and negative associations of different characters with yield and also among themselves. But the nature and extent of contribution of these characters towards yield is not obtained. Path coefficient analysis was used to make partition of the correlation coefficient of the different characters studied to know direct and indirect effects on yield. The information obtained helps in giving proper weightage to the various characters during selection or other breeding program so that the improvement of desirable traits can be achieved effectively. Path coefficient analysis revealed that individual fruit weight (0.704), days to first flowering (0.590), number of fruit cluster (0.259), number of fruits per plant (0.192), days to first harvest (0.107) and AsA (0.0.087) had direct positive effect on yield per plot, indicating these are the main contributors to yield (Table 5). Dhankar et al. [62], Verma and Sarnaik [63], Mageswari [64], Yadav and Singh [65] found that fruits per plant had the highest positive direct effect on yield. The association of AsA content with yield is similar with the findings of Manna and Paul [34]. But days to last harvest $(-0.318)$ was negatively correlated with yield per plant. This negative effect mainly was due to the fact that positive direct effect of days to last harvest on yield was nullified by its negative indirect effects via days to $50 \%$ flowering (-0.176), number of fruit cluster per plant $(-0.053)$, number of primary branches $(-0.061)$ and individual fruit weight (-0.057). However days to $50 \%$ flowering $(-0.792)$, number of primary branches $(-0.169)$, fruit diameter $(-0.055)$, plant height (-0.038), dry matter (\%) (-0.038) and TSS $(-0.038)$ had direct negative effect on yield per plot. Similar results have also been reported by Tiwari and Upadhyay [66] for plant height. But plant height (0.159), fruit diameter (0.105), dry matter (\%) (0.352) and TSS (0.126) were positively correlated with yield per plant. This positive effect mainly was due to the fact that negative direct effect of plant height on yield was nullified by its positive indirect effects via fruits per plant $(-0.091)$, number of primary branches (0.057) and number of fruit cluster (0.048). Negative effect of fruit diameter on yield was nullified by its positive indirect effects via individual fruit weight (0.472). Negative effect of dry matter (\%) on yield was nullified by its positive indirect effects via individual fruit weight $(0.303)$ and days to $50 \%$ flowering (0.172). Negative effect of TSS (-0.038) on yield was nullified by its positive indirect effects via days to $50 \%$ flowering $(0.215)$ and number of fruit cluster $(0.129)$. The residual effect determines how best the causal factors account for the variability of the dependent factor, the yield per plant in this case. In case of the present study the residual effect was 0.41 indicating that the fourteen traits explain only $59 \%$ of variability in yield per plant. The reason seems to be very low and non-significant correlation of some traits with yield. Besides, some other factors which have not been considered here need to be included to account fully for the variation in yield.

The above information revealed that highly significant positive correlation with highest positive direct effect was observed in individual fruit weight followed by number of fruit cluster per plant. Gorbtenko and Gorbtenko [67] and Kumar et al. [69] studied path analysis of economically useful characters of tomato and observed that single fruit weight had an appreciable direct effect on yield per plant. Similar results were also obtained by Alam et al. [68], Kumar et al. [69], Hidayatullah [70] and Islam and Khan [12] in tomato. Previously it was reported that average fruit weight and number of fruits per plant can be used as selection criteria for improvement in tomato [71-73]. So, from this research it may be recommended that the individual fruit weight and number of fruit cluster per plant could be considered as critical criteria for yield improvement in these genotypes of tomatoes.

Table 5. Path-coefficient analysis showing the direct and indirect effect of different yield contributing traits on fruit yield. (Bold diagonal values are direct effects and off-diagonal values are indirect effects)

\begin{tabular}{|c|c|c|c|c|c|c|c|c|c|c|c|c|c|c|}
\hline & $\mathrm{PH}$ & PB & DFF & $50 \% \mathrm{~F}$ & $\mathrm{FC}$ & FH & IFW & FD & FP & LH & DM & TSS & AsA & $\begin{array}{c}\text { Correlation } \\
\text { with YP }\end{array}$ \\
\hline $\mathrm{PH}$ & -0.038 & 0.057 & 0.003 & 0.037 & 0.048 & 0.016 & -0.074 & 0.013 & 0.091 & 0.005 & 0.001 & -0.019 & 0.021 & 0.159 \\
\hline PB & 0.013 & -0.169 & -0.322 & 0.262 & -0.026 & -0.006 & -0.147 & -0.016 & -0.063 & 0.045 & 0.002 & 0.008 & -0.003 & $-0.421 *$ \\
\hline DFF & 0.000 & 0.092 & 0.590 & -0.684 & -0.109 & 0.033 & 0.227 & -0.009 & -0.049 & -0.003 & 0.003 & 0.010 & -0.002 & 0.099 \\
\hline $50 \% \mathrm{~F}$ & 0.002 & 0.056 & 0.510 & -0.792 & -0.143 & 0.036 & 0.125 & -0.010 & -0.062 & 0.028 & 0.008 & 0.010 & -0.008 & -0.240 \\
\hline $\mathrm{FC}$ & -0.007 & 0.017 & -0.249 & 0.438 & 0.259 & -0.001 & -0.115 & 0.021 & 0.078 & -0.031 & 0.001 & -0.019 & 0.004 & $0.396 *$ \\
\hline $\mathrm{FH}$ & -0.006 & 0.009 & 0.184 & -0.265 & -0.002 & 0.107 & 0.038 & -0.002 & -0.011 & 0.003 & 0.006 & -0.006 & 0.022 & 0.078 \\
\hline IFW & 0.004 & 0.035 & 0.190 & -0.141 & -0.042 & 0.006 & 0.704 & -0.037 & -0.085 & -0.010 & -0.016 & 0.009 & -0.008 & $0.609 * * *$ \\
\hline FD & 0.009 & -0.051 & 0.093 & -0.144 & -0.100 & 0.003 & 0.472 & -0.055 & -0.125 & 0.026 & -0.009 & 0.008 & -0.024 & 0.105 \\
\hline FP & -0.018 & 0.055 & -0.151 & 0.255 & 0.105 & -0.006 & -0.311 & 0.036 & 0.192 & -0.034 & 0.001 & -0.013 & 0.017 & 0.126 \\
\hline LH & -0.001 & -0.061 & -0.016 & -0.176 & -0.063 & 0.003 & -0.057 & -0.012 & -0.053 & 0.125 & -0.001 & 0.002 & -0.006 & -0.318 \\
\hline DM & 0.001 & 0.011 & -0.051 & 0.172 & -0.006 & -0.017 & 0.303 & -0.013 & -0.006 & 0.004 & -0.038 & 0.011 & -0.020 & 0.352 \\
\hline TSS & -0.019 & 0.037 & -0.153 & 0.215 & 0.129 & 0.016 & -0.157 & 0.012 & 0.065 & -0.008 & 0.011 & -0.038 & 0.015 & 0.126 \\
\hline AsA & -0.009 & 0.007 & -0.013 & 0.070 & 0.012 & 0.027 & -0.065 & 0.015 & 0.038 & -0.008 & 0.009 & -0.007 & 0.087 & 0.163 \\
\hline
\end{tabular}

Residual effect: 0.41

$*, * *$ and *** indicates significant at $p<0.05, p<0.01$ and $p<0.001$

Here, $\mathrm{PH}=$ Plant height; $\mathrm{PB}=$ Number of primary branches per plant; $\mathrm{DFF}=$ Days to first flowering; $50 \% \mathrm{~F}=\mathrm{Days}$ to $50 \%$ flowering; $\mathrm{FC}=\mathrm{Number}$ of fruit cluster per plant; $\mathrm{FH}=$ Days to first harvest; $\mathrm{IFW}=$ Individual fruit weight; $\mathrm{FD}=$ Fruit diameter; $\mathrm{FP}=\mathrm{Fruits}$ per plant; $\mathrm{YP}=\mathrm{Yield}$ per plant; $\mathrm{LH}=\mathrm{Days}$ to last harvest; DM= Dry matter percentage; TSS= Total soluble solid; AsA = Ascorbic acid content. 


\section{Conclusions and Recommendation}

Plenty of variation was found among the genotypes for the studied traits. Among all the traits number of fruits per plant exhibited high estimates of PCV (3566.48), GCV (3554.12) followed by individual fruit weight $(\mathrm{PCV}=2425.67$, $\mathrm{GCV}=2418.64)$, plant height $(\mathrm{PCV}=543.48, \mathrm{GCV}=521.24)$ and number of fruit cluster per plant $(\mathrm{PCV}=125.15$, GCV=124.51). The lowest PCV (30.54) and GCV (24.59) values were recorded for TSS contents. The studied traits expressed moderate to high heritability estimates ranging from 75.87 to $99.71 \%$. In the present study, high heritability along with high genetic advance was noticed for the traits, individual fruit weight $\left(\mathrm{h}_{\mathrm{b}}^{2}=99.71, \mathrm{GA}=85.40\right)$, fruits per plant $\left(\mathrm{h}_{\mathrm{b}}^{2}=99.65, \mathrm{GA}=81.26\right)$ and plant height $\left(\mathrm{h}_{\mathrm{b}}^{2}=95.61\right.$, $\mathrm{GA}=44.53$ ). These traits can be improved through simple or progeny selection methods. Other traits (i.e. number of primary branches, days to first flowering, days to $50 \%$ flowering, number of fruit cluster per plant, days to first harvest, fruit diameter, yield per plant, days to last harvest, dry matter percentage, total soluble solid and AsA content) showed high heritability along with moderate or low genetic advance which can be improved by inter-mating superior genotypes to develop segregating population for further selection and improvement. Yield per plant was significantly correlated with individual fruit weight $\left(0.609^{* * *}\right)$, number of fruit cluster per plant $\left(0.396^{*}\right)$ in positive direction and significantly negatively correlated with number of primary branch per plant $\left(-0.421^{*}\right)$. The findings suggested that the selection of genotypes having high individual fruit weight with reasonable compromises for higher number of fruit cluster, lower number of primary branches, higher fruit diameter, high number of fruits per plant and moderate plant height should be the priority of breeders to achieve higher yield. Based on our research findings, the genotypes Bahar, Binatomato-7, Binatomato-9, WP10 and Phili-2 can be used for future breeding program targeting yield and quality improvement because these genotypes have the suggested characteristics. The path coefficient analysis revealed that highly significant positive correlation, with highest positive direct effect was found in individual fruit weight $(0.704)$ followed by number of fruit cluster per plant $(0.259)$. Our findings suggest that individual fruit weight and number of fruit cluster per plant could be considered as important criteria for yield improvement in tomato genotypes.

\section{REFERENCES}

[1] J. A. Jenkins. The origin of cultivated tomato, Economic Botany, Vol.2, 379, 1948.

[2] FAO. Food and Agriculture Organization of the United Nations, Production 46 Yearbook 12, Rome, Italy, 2013.

[3] M. Dhaliwal, S. Singh, D. Cheema. Line x tester analysis for yield and processing attributes in tomato, Journal of Research, Vol.40, 49-53, 2003.
[4] P. D. Fraser, S. Romer, C. A. Shipton, P. B. Mills, J. W. Kiano, N. Misawa, R. G. Drake, W. Schuch, P. M. Bramley. Evaluation of transgenic tomato plants expressing an additional phytoene synthase in a fruit-specific manner, Proceedings of the National Academy of sciences, Vol.99, 1092-1097, 2002.

[5] Y. Bai, P. Lindhout. Domestication and breeding of tomatoes: what have we gained and what can we gain in the future?, Annals of Botany, Vol.100, 1085-1094, 2007.

[6] E. Capanoglu, J. Beekwilder, D. Boyacioglu, R. C. De Vos, R. D. Hall. The effect of industrial food processing on potentially health-beneficial tomato antioxidants, Critical reviews in food science and nutrition, Vol.50, 919-930, 2010.

[7] M. Y. Saleem, M. Asghar, Q. Iqbal, A. Rahman, M. Akram. Diallel analysis of yield and some yield components in tomato (Solanum lycopersicum L.), Pakistan Journal of Botany, Vol.45, 1247-1250, 2013.

[8] L. Arab, S. Steck. Lycopene and cardiovascular disease, The American Journal of Clinical Nutrition, Vol.71, 1691s-1695s, 2000.

[9] L. Jarquín-Enríquez, E. Mercado-Silva, J. Maldonado, J. Lopez-Baltazar. Lycopene content and color index of tomatoes are affected by the greenhouse cover, ScientiaHorticulturae, Vol.155, 43-48, 2013.

[10] N. J. Barber, J. Barber. Lycopene and prostate cancer, Prostate Cancer Prostatic Dis., Vol.5, 12, 2002.

[11] J. Shi, M. Le Maguer, M. Bryan. Lycopene from tomatoes. In: Shi, J., Ghazza, Le Maguer, M. (Eds.), Functional Foods. Biochemical and Processing Aspects, Vol.2. CRC Press, Ottawa, Canada, 135-166, 2002.

[12] M. Islam, S. Khan. Variability and character association in tomato (Lycopersicon esculentum Mill.), Bangladesh Journal of Plant Breeding and Genetics, Vol.4, 49-53, 1991.

[13] P. Singh, R. K. Singh, B. C. Saha. Correlation and path analysis in tomato (Lycopersicon esculentum Mill.), Annals of Agricultural Research, Vol.10, 120-124, 1989.

[14] M. E. McGiffen, D. J. Pantone, J. B. Masiunas. Path analysis of tomato yield components in relation to competition with black and eastern black nightshade, Journal of the American Society for Horticultural Science, Vol.119, 6-11, 1994.

[15] N. I. Vavilov. The origin, variation, immunity and breeding of cultivated plants, Soil Science, Vol.72, 482, 1951.

[16] H. Robinson, R. E. Comstock, P. Harvey. Estimates of heritability and the degree of dominance in corn, Agronomy Journal, Vol.20, 125-131, 1949.

[17] H. W. Johnson, H. F. Robinson, R. E. Comstock. Estimation of genetic and environmental variability in soybean, Journal of Agronomy, Vol.47, 314-318, 1955.

[18] D. T. Plummer. An Introduction to Practical Biochemistry, Tata McGraw-Hill Pub.Com: Ltd. Bombay, New Delhi, 1971.

[19] W. D. Hanson. Herilability, Statistical Genetics and Plant Breeding, National Academy of Science, National Research Council, Washington, 125-140, 1961.

[20] G. W. Burton. Quantitative inheritance in grasses, Proc. 6th Int. Grassland Cong 1, 277-283, 1952. 
[21] D. N. Singh, A. Sahu, A. K. Parida. Genetic variability and correlation studies in tomato (Lycopersicon esculentum Mill.), Journal of Environment and Ecology, Vol.15, 117-121, 1997.

[22] R. Allard. 960 Principles of plant breeding. John Wiley and Sons Inc., New York, 1975.

[23] R. Comstock, H. Robinson, J. Gowen. Estimation of average dominance of genes, Heterosis, 494-516, 1952.

[24] P. Miller, J. Williams, H. Robinson, R. Comstock. Estimates of genotypic and environmental variances and covariances in upland cotton and their implications in selection, Agronomy Journal, Vol.50, 126-131, 1958.

[25] S. Wright. Correlation and causation, Journal of Agricultural Research, Vol.20, 557-585, 1921.

[26] D. R. Dewey, K. H. Lu. A correlation and path-coefficient analysis of components of crested wheatgrass seed production, Agronomy Journal, Vol.51, 515-518, 1959.

[27] K. M. Meitei, G. Bora, S. J. Singh, A. K. Sinha. Morphology based genetic variability analysis and identification of important characters for tomato (Solanum lycopersicum L.) crop improvement, Journal of the American Society for Horticultural Science, Vol.86, 114-119, 2014.

[28] S. Mohamed, E. Ali, T. Mohamed. Study of heritability and genetic variability among different plant and fruit characters of tomato (Solanum lycopersicon L.), International Journal of Scientific \& Technology Research, Vol.1, 55-58, 2012.

[29] J. Biswas, S. C. Mallik. Days required for flowering and Harvesting of some promising cultivars of tomato (Lycopersicon esculentum Mill), Environment and Ecology, Vol.7, 1003-1095, 1989.

[30] S. K. Kaushik, D. S. Tomar, A. K. Dixit. Genetics of fruit yield and its contributing characters in tomato (Solanum lycopersicom), Journal of Agricultural Biotechnology and Sustainable Development, Vol.3, 209-213, 2011.

[31] A. Gadekar, L. B. Dhonukshe, F. B. Patil. Studies on variability, heritability and genetic advance in tomato, Journal of Maharashtra Agricultural University, Vol.17, 305-306, 1993.

[32] R. K. Dutta, M. S. Islam, M. Baset, M. A. Majid, M. A. Lahin. Comparative assessment of tomato varieties/advanced lines in relation to growth, yield and quality, Bangladesh Journal of Nuclear Agriculture, Vol.11, 27-35, 1995.

[33] G. Prodan. Some relationships connected with the chemical composition of tomato fruit, Plant Breeding Abstract, Vol.44, 296, 1974.

[34] M. Manna, A. Paul. Studies on genetic variability and characters association of fruit quality parameters in tomato, Hort Flora Research Spectrum, Vol.1, 110-116, 2012.

[35] A. A. Simonov, A. P. Anikeenka, E. E. Yants. Evaluation of tomato varieties for chemical composition, Plant Breeding Abstract, Vol.47, 83, 2001.

[36] J. Tasisa, D. Belew, K. Bantte, W. Gebreselassie. Variability, heritability and Genetic Advance in Tomato (Lycopersicon esculentum Mill.) Genotypes in West Shoa, Ethiopia, American-Eurasian Journal of Agricultural \& Environmental Science, Vol.11, 87-94, 2011.

[37] S. Chernet, D. Belew, F. Abay. Genetic Variability and Association of Characters in Tomato (Solanum lcopersicon L.) Genotypes in Northern Ethiopia, International Journal of Agricultural Research, Vol.8, 67-76, 2013.

[38] O. S. Osekita, A. T. Ademiluyi. Genetic advance, heritability and character association of component of yield in some genotypes of tomato (Lycopersicon esculentum Mill.), Journal of Biotechnology, Vol.2, 6-10, 2014.

[39] K. R. Vinod, K. R. Nandan, S. K.Srivastava,R. Sharma, A. Kumar. Genetic parameters and correlation study for yield and quality traits in tomato (Solanum lycopersicum L.), Plant Archives, Vol.13, 463-467, 2012.

[40] V. V. P. Reddy, K. V. Reddy. Studies in variability in tomato, South Indian Horticulture, Vol.40, 257-260, 1992.

[41] S. Shashikanth, N. Basavaraj, R. Hosamani, B. Patil. Genetic variability in tomato (Solanum lycopersicon [Mill]. Wettsd.), Karnataka Journal of Agricultural Sciences, Vol.23, 536-537, 2010 .

[42] M. S. Kumar, A. K. Pal, A. K. Singh, K. Sati, D. Kumar. Studies on genetic parameters to improve the genetic architecture of tomato (Solanum lycopersicum L.), International Journal of Applied Biology and Pharmaceutical Technology, Vol.4, 234-237, 2013.

[43] R. A. Dar, J. P. Sharma. Genetic variability studies of yield and quality traits in tomato (Solanum lycopersicum L.), International Journal of Plant Breeding and Genetics, Vol.5, 168-174, 2011.

[44] I. Golani, D. Mehta, V. Purohit, H. Pandya, M. Kanzariya. Genetic variability, correlation and path coefficient studies in tomato, Indian Journal of Agricultural Research, Vol.41, 146, 2007.

[45] O. P. Meena, V. Bahadur. Assessment of genetic variability, heritability and genetic advance among tomato (Solanum lycopersicum L.) germplasm, Agricultural Science Digest, Vol.27, 185-192, 2014a.

[46] N. Mehta, B. Asati. Genetic relationship of growth and development traits with fruit yield in tomato (Lycopersicon esculentum Mill), Karnataka Journal of Agricultural Sciences, Vol.21, 2010.

[47] J. Tasisa, D. Belew, K. Bantte. Genetic associations analysis among some traits of tomato (Lycopersicon esculentum Mill.) Genotypes in West Showa, Ethiopia, International Journal of Plant Breeding \& Genetics, Vol.6, 129-139, 2012.

[48] B. K. Mohanty. Studies on variability, heritability, interrelationship and path analysis in tomato, Annals of Agricultural Research, Vol.23, 65-69, 2002.

[49] P. C. Singh, R. Kumar, M. Singh, A. Rai, M. Singh, M. Rai. Identification of resistant sources against early blight disease of tomato, Indian Journal of Horticulture, Vol.68, 516-521, 2011.

[50] F. Al-Aysh, M. Al-Serhan, A. Al-Shareef, M. Al-Nasser, H. Kutma. Study of genetic parameters and character interrelationship of yield and some yield components in tomato (Solanum lycopersicum L.), International Journal of Genetics, Vol.2, 29-33, 2012.

[51] A. Shankar, R. Reddy, M. Sujatha, M. Pratap. Genetic variability studies in F1 generation of tomato (Solanum lycopersicon L.), IOSR Journal of Agriculture and Veterinary Science, Vol.4, 31-34, 2013.

[52] S. Barooah, N. K. Mohan. Correlation study between fruit size and ascorbic acid content in tomato, Current Research Bangalore India, Vol.5, 82, 1976.

[53] Z. Susic, N. Pavlovic, D. Cvikic, T. Sretenovic-Rajicic. Studies of correlation between yield and fruit characteristics of (Lycopersicon esculentum Mill.) hybrids and their parental genotypes, In "II Balkan Symposium on Vegetables and Potatoes, Vol.579, 163-166, 2000.

[54] C. I. Rani, I. Muthuvel, D. Veeraragavathatham. Correlation 
and path coefficient for yield components and quality traits in tomato (Lycopersicon esculentum Mill.), Agricultural Science Digest, Vol.30, 11-14, 2010.

[55] B. R. Reddy, M. P. Reddy, D. S. Reddy, H. Begum. Correlation and path analysis studies for yield and quality traits in tomato (Solanum lycopersicum L.), IOSR Journal of Agriculture and Veterinary Science, Vol.4, 56-59, 2013.

[56] M. Kumar, B. S. Dudi. Study of correlation for yield and quality characters in tomato (Lycopersicon esculentum Mill.), Electronic Journal of Plant Breeding, Vol.2, 453-460, 2011.

[57] B. Sharma, J. P. Singh. Correlation and path coefficient analysis for quantitative and qualitative traits for fruit yield and seed yield in tomato genotypes, Indian Journal of Horticulture, Vol.69, 540-544, 2012.

[58] B. M. R. Islam, N. A. Ivy, M. G. Rasul, M. Zakaria. Character association and path analysis of exotic tomato (Solanum lycopersicum L.) genotypes, Bangladesh Journal of Plant Breeding and Genetics, Vol.23, 13-18, 2010.

[59] L. M. de Souza, P. C. T. Melo, R. R. Luders, A. M. Melo. Correlations between yield and fruit quality characteristics of fresh market tomatoes, Horticultura Brasileira, Vol.30, 627-631, 2012.

[60] P. Anitha, R. R. Sharma, R. N. Tiwari, A. K. Sureja. Correlation and path analysis for some horticultural traits in tomato, Indian Journal of Horticulture, Vol.64, 101-109, 2007.

[61] O. P. Meena, V. Bahadur. Assessment of correlation and path coefficient analysis for yield and yield contributing traits among tomato (Solanum lycopersicum L.) germplasm, Agricultural Science Digest, Vol.34, 245-250, 2014 b.

[62] S. Dhankar, B. Dhankhar, N. Sharma. Correlation and path analysis in tomato under normal and high temperature conditions, Haryana Journal of Horticultural Sciences, Vol.30, 89-91, 2001.

[63] S. K. Verma, D. A. Sarnaik. Path analysis of yield components in tomato (Lycopersicon esculentum Mill),
Journal of Applied Biology, Vol.10, 136-138, 2000.

[64] K. Mageswari, S. Natarajan, S. Thamburaj. Causal influence of component traits on yield in hybrids of tomato, Journal of South Indian Horticulture, Vol.47, 179-181, 1999.

[65] D. S. Yadav, S. P. Singh. Correlation and path analysis in tomato, Journal of Hill Research, Vol.11, 207-221, 1998.

[66] J. K. Tiwari, D. Upadhyay. Correlation and path coefficient studies in tomato (Lycopersicon esculentum Mill.), Agricultural Science Research Journal, Vol.2, 63-38, 2011

[67] E. M. Gorbtenko, I. Y. U. Gorbtenko. Path analysis of economically useful characters in tomato, Tsitologiya Genetika, Vol.19, 206-210, 1985.

[68] M. Alam, Q. Ahmed, M. Ali. Correlation and path coefficient analysis for some characters in tomato, Bangladesh Journal of Plant Breeding and Genetics, Vol.3, 34-44, 1988.

[69] D. Kumar, R. Kumar, S. Kumar, M. L. Bhardwaj, M. C. Thakur, R. Kumar, K. S. Thakur, B. S. Dogra, A. Vikram, A. Thakur, P. Kumar. Genetic variability, Correlation and path coefficient analysis in Tomato, International Journal of Vegetable Science, Vol.19, 313-323, 2013.

[70] Hidayatullah, S. A. Jjatoi, A. Ghafoor.,T. Mahmood. Path coefficient analysis of yield component in Tomato (Lycopersicon esculentum), Pakistan Journal of Botany, Vol. 40, 627-635, 2008.

[71] R. Kumar, C. N. Ram, G. C. Yadav, C. Deo, S. C. Vimal, H. D. Bhartiya. Studies on correlation and path coefficient analysis in tomato (Solanum lycopersicon L.), Plant Archives, Vol. 14, 443-447, 2014.

[72] P. K. Singh, B. Singh, S. Pandey. Genetic Variability and Character Association Analysis in Tomato, Indian Institute of Vegetable Research, Vol. 19, 2006.

[73] T. Buckseth, M. K. Sharma, K. S. Thakur. Genetic diversity and path analysis in tomato (Solanum lycopersicum L.), Vegetable Science, Vol. 39, 221-223, 2012. 